\title{
Robust Two-Stage Stochastic Linear Programs with Moment Constraints *
}

\author{
Sarah Y. Gao ${ }^{\dagger}$ Lingchen Kong ${ }^{\ddagger}$ Jie $\mathrm{Sun}^{\S}$
}

April 30, 2013, Revised Feb 10, 2014

\begin{abstract}
.
We consider the two-stage stochastic linear programming model, in which the recourse function is a worst case expected value over a set of probabilistic distributions. These distributions share the same first and second order moments. By using duality of semi-infinite programming and assuming knowledge on extreme points of the dual polyhedron of the constraints, we show that a deterministic equivalence of the two-stage problem is a second-order cone optimization problem. Numerical examples are presented to show non-conservativeness and computational advantage of this approach.
\end{abstract}

Key words. Second order cone optimization; Stochastic programming

MSC2010. 90B50, 90C15

${ }^{*}$ This paper is dedicated to Professor Minyi Yue of Chinese Academy of Science in celebration of his 90th birthday. Research is partially supported by The Provost's Chair Grant at National University of Singapore, the National Basic Research Program of China (2010CB732501), and the National Natural Science Foundation of China (11171018).

${ }^{\dagger}$ Department of Decision Sciences, National University of Singapore. Email: yini.gao@nus.edu.sg

${ }^{\ddagger}$ Department of Mathematics, Beijing Jiaotong University. Email: konglchen@126.com

$\S^{\S}$ Department of Decision Sciences, National University of Singapore and Department of Mathematics and Statistics, Curtin University, Australia. Email: jsun@nus.edu.sg 


\section{Introduction}

Consider the following two-stage stochastic programming problem with fixed recourse:

$$
\min _{x \in X}\left\{c^{\prime} x+\mathbb{E}_{\mathbb{P}}[Q(x, \tilde{z})]\right\}
$$

where the apostrophe $\left({ }^{\prime}\right)$ stands for the transpose and

$$
\begin{aligned}
Q(x, \tilde{z})=\min & d^{\prime} y \\
\text { s.t. } & A x+D y=\tilde{z}, \\
& y \geq 0
\end{aligned}
$$

where $x \in \mathbb{R}^{n}$ is the vector of first-stage decision variables subject to a feasible region $X=\{x$ : $B x=b, x \geq 0\} \subseteq \mathbb{R}^{n}, B \in \mathbb{R}^{q \times n}, b \in \mathbb{R}^{q}$, while $d \in \mathbb{R}^{k}, A \in \mathbb{R}^{l \times n}$ and $D \in \mathbb{R}^{l \times k}$ are fixed second-stage data; in particular, $D$ is called the fixed recourse matrix. Finally, $\tilde{z} \in \mathbb{R}^{l}$ is random vector with a support $\Omega \subset \mathbb{R}^{l}, \mathbb{P}$ is the probability measure of $\tilde{z}$, and $\mathbb{E}_{\mathbb{P}}$ is expectation under probability measure $\mathbb{P}$.

Practically speaking, it is rare to know the exact distribution of $\tilde{z}$, but we often know the bounds of means and variances of the components of $\tilde{z}$ by collecting historical data. For convenience, assume that we do not know the exact probability measure of $\tilde{z}$, but we are given $\mathbb{E}_{\mathbb{P}}\left(\tilde{z}_{i}\right) \leq \mu_{i}$ and $\mathbb{E}_{\mathbb{P}}\left(\tilde{z}_{i}^{2}\right) \leq \eta_{i}$ for $i=1, \ldots, l$. Motivated by recent development in robust optimization, say Ben-Tal and Nemirovski (2002) and Bertsimas et al.(2010), we consider the "robust version" of the Problem (1.1) (also called the "minimax two-stage stochastic programs"),

$$
\min _{x \in X}\left\{c^{\prime} x+\max _{\mathbb{P} \in \mathcal{F}} \mathbb{E}_{\mathbb{P}}[Q(x, \tilde{z})]\right\}
$$

where

$$
\mathcal{F}:=\left\{\mathbb{P}: \mathbb{P}(\tilde{z} \in \Omega)=1, \mathbb{E}_{\mathbb{P}}\left(\tilde{z}_{j}\right) \leq \mu_{j}, \mathbb{E}_{\mathbb{P}}\left(\tilde{z}_{j}^{2}\right) \leq \eta_{j}, \quad j=1, \ldots, l\right\},
$$

In a recent paper, Ang et al. (2014) considered a similar problem with an additional assumption on $y$, which requires that $y$ is affinely dependent on $z$. We call this type of assumptions "the linear decision rule" following Ben-Tal and Nemirovski (2002). The difference in this paper is that we do not use linear decision rule, instead, we assume that we have sufficient knowledge on the following dual polyhedron

$$
\left(\begin{array}{ll}
A & D \\
B & 0
\end{array}\right)^{\prime}\left(\begin{array}{l}
w \\
u
\end{array}\right) \leq\left(\begin{array}{l}
c \\
d
\end{array}\right)
$$

namely, assume that

Assumption 1 We know all the extreme points of the set

$$
\left(\begin{array}{ll}
A & D \\
B & 0
\end{array}\right)^{\prime}\left(\begin{array}{l}
w \\
u
\end{array}\right) \leq\left(\begin{array}{l}
c \\
d
\end{array}\right)
$$


Let us for convenience of analysis denote these extreme points by

$$
\left(\begin{array}{c}
w^{i} \\
u^{i}
\end{array}\right), i=1, \ldots, m
$$

The rest of this paper is organized as follows. In Section 2, we establish the deterministic equivalence of Problem (1.2) as a second-order cone (SOC for short) optimization problem. In Section 3, we present numerical results with certain important observations. Section 4 concludes the paper.

Notations. We denote a random variable, $\tilde{z}$, with the tilde sign. Matrices and vectors are represented as upper and lower case letters respectively. If $x$ is a vector, we use the notation $x_{i}$ to denote the $i$ th component of the vector. For any two vectors $x, y \in \mathbb{R}^{l}$, the notation $x \leq y$ means $x_{i} \leq y_{i}$ for all $i=1, \ldots, l$.

\section{Problem Formulation}

Consider problem

$$
\min _{x \in X}\left\{c^{\prime} x+\max _{\mathbb{P} \in \mathcal{F}} \mathbb{E}_{\mathbb{P}}[Q(x, \tilde{z})]\right\}
$$

where

$$
\begin{aligned}
Q(x, \tilde{z})=\min & d^{\prime} y \\
\text { s. t. } & A x+D y=\tilde{z}, \\
& y \geq 0 .
\end{aligned}
$$

and

$$
\mathcal{F}:=\left\{\mathbb{P}: \mathbb{P}(\tilde{z} \in \Omega)=1, \mathbb{E}_{\mathbb{P}}\left(\tilde{z}_{j}\right) \leq \mu_{j}, \mathbb{E}_{\mathbb{P}}\left(\tilde{z}_{j}^{2}\right) \leq \eta_{j}, \quad j=1, \ldots, l\right\},
$$

and $\Omega=\left\{z \in \mathbb{R}^{l}:-\infty \leq-\ell \leq z \leq h \leq+\infty\right\}$. Since $x$ is independent of $\mathbb{P}$, Problem (2.4) can be re-written as follows:

$$
\min _{x \in X} \max _{\mathbb{P} \in \mathcal{F}} \mathbb{E}_{\mathbb{P}}\left[c^{\prime} x+Q(x, \tilde{z})\right]
$$

We need the following assumptions.

\section{Assumption 2}

$$
\min _{x \in X} \max _{\mathbb{P} \in \mathcal{F}} \mathbb{E}_{\mathbb{P}}\left[c^{\prime} x+Q(x, \tilde{z})\right]=\max _{\mathbb{P} \in \mathcal{F}} \min _{x \in X} \mathbb{E}_{\mathbb{P}}\left[c^{\prime} x+Q(x, \tilde{z})\right]
$$




\section{Assumption 3}

$$
\min _{x \in X}\left\{\mathbb{E}_{\mathbb{P}}\left[c^{\prime} x+Q(x, \tilde{z})\right]\right\}=\mathbb{E}_{\mathbb{P}}\left\{\min _{x \in X}\left[c^{\prime} x+Q(x, \tilde{z})\right]\right\}
$$

Assumption 2 is valid if $X$ is compact. Otherwise, there are several other conditions to guarantee the min-max relationship above, see Sion (1958). A general result about Assumption 3 can be found in Rockafellar and Wets (1998)(Theorem 14.60) as follows. Let $\mathcal{X}$ be a space of measurable functions from $T$ to $\mathbb{R}^{n}$ that is decomposable relative to $\mu$, a $\sigma$-finite measure on $\mathcal{A}$. Let $f: T \times \mathbb{R}^{n} \rightarrow \overline{\mathbb{R}}$ be a normal integrand. Then the minimization of $I_{f}$ over $\mathcal{X}$ can be reduced to pointwise minimization in the sense that, as long as $I_{f} \neq \infty$ on $\mathcal{X}$, one has

$$
\inf _{x \in \mathcal{X}} \int_{T} f(t, x(t)) \mu(d t)=\int_{T}\left[\inf _{x \in \mathbb{R}^{n}} f(x, t)\right] \mu(d t) .
$$

Moreover, as long as this common value is not $-\infty$ on $\mathcal{X}$, one has for $\bar{x} \in \mathcal{X}$ that

$$
\bar{x} \in \operatorname{argmin}_{x \in \mathcal{X}} I f(x) \Longleftrightarrow \bar{x}(t) \in \operatorname{argmin}_{x \in \mathbb{R}^{n}} f(t, x) \text { for } \mu \text {-almost every } t \in T \text {. }
$$

Under Assumptions 1-3, we have that Problem (2.4) is equivalent to

$$
\begin{aligned}
& \max _{\mathbb{P} \in \mathcal{F}} \min _{x \in X}\left\{c^{\prime} x+\max _{\mathbb{P} \in \mathcal{F}} \mathbb{E}_{\mathbb{P}}[Q(x, z)]\right\} \\
& =\quad \max _{\mathbb{P} \in \mathcal{F}} \min _{x \in X} \mathbb{E}_{\mathbb{P}}\left[\min _{y}\left\{c^{\prime} x+d^{\prime} y:\left(\begin{array}{cc}
A & D \\
B & 0
\end{array}\right)\left(\begin{array}{l}
x \\
y
\end{array}\right)=\left(\begin{array}{l}
z \\
b
\end{array}\right), x, y \geq 0\right\}\right] \\
& =\max _{\mathbb{P} \in \mathcal{F}} \mathbb{E}_{\mathbb{P}} \min _{x \in X}\left[\min _{y}\left\{c^{\prime} x+d^{\prime} y:\left(\begin{array}{cc}
A & D \\
B & 0
\end{array}\right)\left(\begin{array}{l}
x \\
y
\end{array}\right)=\left(\begin{array}{l}
z \\
b
\end{array}\right), x, y \geq 0\right\}\right] \\
& =\max _{\mathbb{P} \in \mathcal{F}} \mathbb{E}_{\mathbb{P}} \max _{1 \leq i \leq m}\left(z^{\prime} w^{i}+b^{\prime} u^{i}\right) \\
& =\min \left\{v_{0}+z^{\prime} v+\eta^{\prime} V: v_{0}+z^{\prime} v+\left(z^{2}\right)^{\prime} V \geq z^{\prime} w^{i}+b^{\prime} u^{i}, 1 \leq i \leq m, z \in \Omega\right\}
\end{aligned}
$$

where $z^{2}:=\left(z_{1}^{2}, \ldots, z_{\ell}^{2}\right)^{\prime}$; the first two equalities above are due to Assumption 2 and Assumption 3 , respectively. The second last equality is due to Assumption 1, and the last equality is due to strong duality of general linear programming (assume it holds ${ }^{1}$ ), which should be clear if we note that the following is a pair of general dual linear programs.

$$
\begin{aligned}
& \max _{\mathbb{P}} \mathbb{E}_{\mathbb{P}}\left[\max _{1 \leq i \leq m}\left(z^{\prime} w^{i}+b^{\prime} u^{i}\right)\right] \\
& \text { s.t. } \quad \mathbb{E}_{\mathbb{P}}(\Omega)=1 \\
& E_{\mathbb{P}}\left(z_{i}\right) \leq \mu_{i}, i=1, \ldots, l \\
& E_{\mathbb{P}}\left(z_{i}^{2}\right) \leq \eta_{i}, i=1, \ldots, l .
\end{aligned}
$$

Proposition 2.1 The robust stochastic optimization problem (2.4) is equivalent to the following second-order cone (SOC for short) optimization problem

$$
\min _{v_{0}, v, V, r, \lambda, \nu} v_{0}+\mu^{\prime} v+\eta^{\prime} V
$$

\footnotetext{
${ }^{1}$ The conditions ensuring such strong duality can be found in Anderson and Nash (1987).
} 


$$
\begin{array}{ll}
\text { s. t. } & \sum_{j=1}^{l}\left[h_{j} \lambda_{j}^{i}+\ell_{j} \nu_{j}^{i}+u_{j}^{i}\right]-v_{0}+b^{\prime} u^{i} \leq 0, i=1, \ldots, m, \\
& \left\|\left(\begin{array}{c}
v_{j}-w_{j}^{i}+\lambda_{j}^{i}-\nu_{j}^{i} \\
V_{j}-r_{j}^{i}
\end{array}\right)\right\| \leq V_{j}+r_{j}^{i}, j=1, \ldots, l, i=1, \ldots, m, \\
& V_{j}, v_{j} r_{j}^{i}, \lambda_{j}^{i}, \nu_{j}^{i} \geq 0, j=1, \ldots, l, i=1, \ldots, m .
\end{array}
$$

Proof. The feasible set of (2.5) can be equivalently written as

$$
\begin{aligned}
& \left(v_{0}-b^{\prime} u^{i}+\left(v-w^{j}\right)^{\prime} z+\sum_{j=1}^{l} V_{j} z_{j}^{2}\right) \geq 0, \forall i=1, \ldots, m, z \in \Omega, \\
& V \geq 0, v \geq 0 .
\end{aligned}
$$

For fixed $i$, the first constraint above is equivalent to the following

$$
\min _{z}\left(v_{0}-b^{\prime} u^{i}+\left(v-w^{i}\right)^{\prime} z+\sum_{j=1}^{l} V_{j} z_{j}^{2}:-\ell \leq z \leq h\right) \geq 0
$$

Fix $V_{j}, v_{j}, v_{0}, u^{i}, w^{i}$, the left hand side of (2.6) is a separable convex quadratic program in $z$ over a box. By strong duality of convex quadratic programming and the separability of variables, we have that (2.6) is equivalent to

$$
\begin{array}{ll}
\max & \sum_{j=1}^{l}\left[-h_{j} \lambda_{j}-\ell_{j} \nu_{j}+V_{j} z_{j}^{2}+\left(v_{j}-w_{j}^{i}+\lambda_{j}-\nu_{j}\right) z_{j}\right]+v_{0}-b^{\prime} u^{i} \geq 0 \\
\text { s.t. } & V_{j}, v_{j}, \lambda_{j}, \nu_{j} \geq 0, j=1, \ldots, l, \\
& 2 V_{j} z_{j}+\left(v_{j}-w_{j}^{i}+\lambda_{j}-\nu_{j}\right)=0, j=1, \ldots, l,
\end{array}
$$

where $\lambda_{j}, \nu_{j}$ are dual variables.

If all $V_{j}>0$, we solve $z_{j}$ from (2.8) and substitute the solution into (2.7) to obtain

$$
\begin{array}{ll}
\max & \sum_{j=1}^{m}\left[-h_{j} \lambda_{j}-\ell_{j} \nu_{j}-\left(v_{j}-w_{j}^{i}+\lambda_{j}-\nu_{j}\right)^{2} /\left(4 V_{j}\right)\right]+v_{0}-b^{\prime} u^{i} \geq 0 \\
\text { s. t. } & V_{j}, v_{j}, \lambda_{j}, \nu_{j} \geq 0, j=1, \ldots, l,
\end{array}
$$

which is equivalent to

$$
\begin{aligned}
& \sum_{j=1}^{l}\left[-h_{j} \lambda_{j}-\ell_{j} \nu_{j}-r_{j}\right]+v_{0}-b^{\prime} u^{i} \geq 0, \\
& V_{j}, v_{j}, r_{j}, \lambda_{j}, \nu_{j} \geq 0, j=1, \ldots, l, \\
& \left(v_{j}-w_{j}^{i}+\lambda_{j}-\nu_{j}\right)^{2} \leq 4 V_{j} r_{j}^{i}, j=1, \ldots, l,
\end{aligned}
$$

where $r_{j}$ s are auxiliary variables. 
If some $V_{j}=0$, it can be directly verified that conditions (2.9)-(2.11) are also sufficient and necessary for the optimality of Problem (2.7)-(2.8). Thus, (2.4) is equivalent to

$$
\begin{array}{cl}
\min _{r, v_{0}, v, V, \lambda, \nu} & v_{0}+\mu^{\prime} v+\eta^{\prime} V \\
\text { s.t. } & \sum_{j=1}^{l}\left[-h_{j} \lambda_{j}-\ell_{j} \nu_{j}-r_{j}^{i}\right]+v_{0}-b^{\prime} u^{i} \geq 0, j=1, \ldots, l, i=1, \ldots, m \\
& \left(v_{j}-w_{j}^{i}+\lambda_{j}-\nu_{j}\right)^{2} \leq 4 V_{j} r_{j}^{i}, j=1, \ldots, l, i=1, \ldots, m \\
& V, v, \lambda, \nu, r \geq 0 .
\end{array}
$$

The problem is an SOC optimization problem since we can reformulate it as follows.

$$
\begin{aligned}
\min _{v_{0}, v, V, r \lambda, \nu} & v_{0}+\mu^{\prime} v+\eta^{\prime} V \\
\text { s. t. } & \sum_{j=1}^{l}\left[h_{j} \lambda_{j}^{i}+\ell_{j} \nu_{j}^{i}+r_{j}^{i}\right]-v_{0}+b^{\prime} u^{i} \leq 0, j=1, \ldots, l, i=1, \ldots, m, \\
& \left\|\left(\begin{array}{c}
v_{j}-w_{j}^{i}+\lambda_{j}^{i}-\nu_{j}^{i} \\
V_{j}-r_{j}^{i}
\end{array}\right)\right\| \leq V_{j}+r_{j}^{i}, j=1, \ldots, l, i=1, \ldots, m, \\
& V_{j}, v_{j} r_{j}^{i}, \lambda_{j}^{i}, \nu_{j}^{i} \geq 0, j=1, \ldots, l, i=1, \ldots, m .
\end{aligned}
$$

Remarks. In case that $\ell=-\infty$, or $h=+\infty$ or both, the format of the SOC optimization problem could be slightly different, but it does not change the essential point that Problem (2.4) is equivalent to an SOC problem.

The right hand side of the second-stage constraints are not necessarily $\tilde{z}$. In fact it can be an affine mapping of $\tilde{z}$ without essential change of the conclusion.

\section{$3 \quad$ Numerical Experiments}

\subsection{A Classic Example}

Example. ${ }^{2}$ A company manager is considering the amount of steel to purchase (at $\$ 58 / 1000 \mathrm{lb}$ ) for producing wrenches and pliers in next month. The manufacturing process requires molding the tools on a molding machine and then assembling the tools on an assembly machine with the following technical data.

\begin{tabular}{l|cc} 
& Wrench & Plier \\
\hline Steel (lbs.) & 1.5 & 1 \\
Molding Machine (hours) & 1 & 1 \\
Assembly Machine (hours) & .3 & .5 \\
Contribution to Earnings (\$/1000 units) & 130 & 100
\end{tabular}

\footnotetext{
${ }^{2}$ This is a slightly different version of Example 7.3 in the book of Bertsimas and Freund (2000).
} 
There are uncertainties that will influence his decision. 1. The total available assembly hours of next month could be 8000 or 10,000, with 50/50 chance. 2 . The total available molding hours of next month could be either 21,000 or 25,000 at $50 \%$ possibility for each case. The manager would like to plan, in addition to the amount of steel to purchase, for the production of wrenches and pliers of next month so as to maximize the expected net revenue of this company.

\subsection{The Two-stage Stochastic Programming Formulation}

Decision Variables: $x$ : the steel to purchase now; $w_{i}, p_{i}$ : production plan under scenario $i=$ $1,2,3,4$.

\begin{tabular}{c|ccc} 
Scenario & Assembly Hours & Molding Hours & Probability \\
\hline 1 & 8000 & 25000 & .25 \\
2 & 8000 & 21000 & .25 \\
3 & 10000 & 25000 & .25 \\
4 & 10000 & 21000 & .25
\end{tabular}

We solve the problem as a traditional two-stage stochastic programming problem (i.e. in format (1.1)) as follows.

$$
\begin{array}{ccl}
\min & 58 x-\sum_{i=1}^{4} .25\left(130 w_{i}+100 p_{i}\right) & \\
\text { s.t. } & w_{1}+p_{1} \leq 25000 & \text { (Mold constraint for scenario 1) } \\
.3 w_{1}+.5 p_{1} \leq 8000 & \text { (Assembly constraint for scenario 1) } \\
-x+1.5 w_{1}+p_{1} \leq 0 & \text { (Steel constraint for scenario 1) } \\
w_{2}+p_{2} \leq 21000 & \text { (Mold constraint for scenario 2) } \\
.3 w_{2}+.5 p_{2} \leq 8000 & \text { (Assembly constraint for scenario 2) } \\
-x+1.5 w_{2}+p_{2} \leq 0 & \text { (Steel constraint for scenario 2) } \\
w_{3}+p_{3} \leq 25000 & \text { (Mold constraint for scenario 3) } \\
.3 w_{3}+.5 p_{3} \leq 10000 & \text { (Assembly constraint for scenario 3) } \\
-x+1.5 w_{3}+p_{3} \leq 0 & \text { (Steel constraint for scenario 3) } \\
w_{4}+p_{4} \leq 21000 & \text { (Mold constraint for scenario 4) } \\
.3 w_{4}+.5 p_{4} \leq 10000 & \text { (Assembly constraint for scenario 4) } \\
-x+1.5 w_{4}+p_{4} \leq 0 & \text { (Steel constraint for scenario 4) } \\
x, w_{i}, p_{i} \geq 0, i=1, \ldots, 4 . &
\end{array}
$$

The optimal cost $=-961.89$. 
We next solve it as a robust two-stage stochastic programming problem (i.e. in format (2.5)). Here we have $X=\{x: x \geq 0\},(B, b)=\emptyset$ and

$$
\begin{gathered}
c=58, d=\left[\begin{array}{c}
-130 \\
-100 \\
0 \\
0
\end{array}\right], y=\left[\begin{array}{c}
w \\
p \\
\tau_{1} \\
\tau_{2}
\end{array}\right], A=\left[\begin{array}{c}
0 \\
0 \\
-1
\end{array}\right] \\
D=\left[\begin{array}{cccc}
1 & 1 & 1 & 0 \\
.3 & .5 & 0 & 1 \\
1.5 & 1 & 0 & 0
\end{array}\right], \tilde{z}=\left[\begin{array}{c}
\tilde{z}_{1} \\
\tilde{z}_{2} \\
\tilde{z}_{3}
\end{array}\right], \ell=\left[\begin{array}{c}
-21 \\
-8 \\
1
\end{array}\right], h=\left[\begin{array}{c}
25 \\
10 \\
1
\end{array}\right],
\end{gathered}
$$

where $\tau_{1}, \tau_{2}$ are slack variables, $\tilde{z}_{3} \equiv 0$, and $\tilde{z}_{1}, \tilde{z}_{2}$ are independent random variables with

$$
\begin{aligned}
& \mathbb{P}\left(\tilde{z}_{1}=21\right)=\mathbb{P}\left(\tilde{z}_{1}=25\right)=\mathbb{P}\left(\tilde{z}_{2}=8\right)=\mathbb{P}\left(\tilde{z}_{2}=10\right)=.5 \\
& \mathbb{E}\left(\tilde{z}_{1}\right)=23, \mathbb{E}\left(\tilde{z}_{2}\right)=9, \mathbb{E}\left(\tilde{z}_{3}\right)=0 .
\end{aligned}
$$

We have $\mathbb{E}\left(\tilde{z}_{1}^{2}\right)=533, \mathbb{E}\left(\tilde{z}_{2}^{2}\right)=82, \mathbb{E}\left(\tilde{z}_{3}^{2}\right)=0$.

We calculate the extreme points of the feasible region of the dual problem, that is,

$$
\left\{w \in \mathbb{R}^{3} \mid D^{\prime} w \leq d, A^{\prime} w \leq c\right\},
$$

where $D, A, c$, and $d$ are given as above. The extreme points of (3.12) are

$$
w^{1}=\left(\begin{array}{c}
-43 \\
0 \\
-58
\end{array}\right), \quad w^{2}=\left(\begin{array}{c}
0 \\
-143.3 \\
-58
\end{array}\right)
$$

We then solve the corresponding SOC optimization problem:

$$
\begin{array}{cl}
\min _{v_{0}, v, V, r, \lambda, \nu} & v_{0}+23 v_{1}+9 v_{2}+533 V_{1}+82 V_{2} \\
\text { s.t. } & -v_{0}+\sum_{j=1}^{3} r_{j}^{1}+25 \lambda_{1}^{1}+10 \lambda_{2}^{1}+\lambda_{3}^{1}-21 \nu_{1}^{1}-8 \nu_{2}^{1}+\nu_{3}^{1} \leq 0 \\
& -v_{0}+\sum_{j=1}^{3} r_{j}^{2}+25 \lambda_{1}^{2}+10 \lambda_{2}^{2}+\lambda_{3}^{2}-21 \nu_{1}^{2}-8 \nu_{2}^{2}+\nu_{3}^{2} \leq 0 \\
& \left\|\left(\begin{array}{c}
v_{1}+\lambda_{1}^{1}-\nu_{1}^{1}+43 \\
V_{1}-r_{1}^{1}
\end{array}\right)\right\| \leq V_{1}+r_{1}^{1} \\
& \left\|\left(\begin{array}{c}
v_{2}+\lambda_{2}^{1}-\nu_{2}^{1} \\
V_{2}-r_{2}^{1}
\end{array}\right)\right\| \leq V_{2}+r_{2}^{1} \\
& \left\|\left(\begin{array}{c}
v_{3}+\lambda_{3}^{1}-\nu_{3}^{1}+58 \\
V_{3}-r_{3}^{1}
\end{array}\right)\right\| \leq V_{3}+r_{3}^{1} \\
& \left\|\left(\begin{array}{c}
v_{1}+\lambda_{1}^{2}-\nu_{1}^{2} \\
V_{2}-r_{1}^{2}
\end{array}\right)\right\| \leq V_{1}+r_{1}^{2}
\end{array}
$$




$$
\begin{aligned}
& \left\|\left(\begin{array}{c}
v_{2}+\lambda_{2}^{2}-\nu_{2}^{2}+143.38 \\
V_{2}-r_{2}^{2}
\end{array}\right)\right\| \leq V_{2}+r_{2}^{2} \\
& \left\|\left(\begin{array}{c}
v_{3}+\lambda_{3}^{2}-\nu_{3}^{2}+58 \\
V_{3}-r_{3}^{2}
\end{array}\right)\right\| \leq V_{3}+r_{3}^{2} \\
& V_{i}, v_{i}, r_{j}^{i}, \lambda_{j}^{i}, \nu_{j}^{i} \geq 0, i=1,2,3 ; j=1,2 .
\end{aligned}
$$

The numerical results are as follows. Optimal cost $=-902.89$, and

$$
\begin{gathered}
v_{0}=-903, v=\left(\begin{array}{l}
0.0007 \\
0.0017 \\
0.5579
\end{array}\right), V=\left(\begin{array}{c}
0 \\
0 \\
11311
\end{array}\right), \\
r^{1}=\left(\begin{array}{l}
0.0388 \\
0.0346 \\
0.0777
\end{array}\right), r^{2}=\left(\begin{array}{l}
36.8178 \\
36.9124 \\
55.9710
\end{array}\right), \lambda^{1}=\left(\begin{array}{c}
0.0004 \\
0.0008 \\
0.0015
\end{array}\right), \lambda^{2}=\left(\begin{array}{c}
9.3722 \\
9.9876 \\
18.8041
\end{array}\right), \\
\nu^{1}=\left(\begin{array}{c}
43.0059 \\
0.0103 \\
0.0015
\end{array}\right), \nu^{2}=\left(\begin{array}{c}
9.3765 \\
153.3265 \\
18.9580
\end{array}\right) .
\end{gathered}
$$

Ang et al.(2014) tested the same problem based on the linear decision rule mentioned in Section 1 and obtained an optimal cost $-900.9^{3}$. Compared to the numerical results obtained in this paper, we can see that the solution (optimal cost $=-961.89$ ) of the classical two-stage model with exact distribution is less conservative than the model studied in this paper (optimal cost $=-900.90)$. Our solution is a bit conservative $(7 \%$ worse) but not overly conservative. In addition, our result is slightly better that the case of using linear decision rule (LDR). We guess the reason was that LDR is a restrictive assumption and thus reduces the size of feasible region. The optimal value is then more conservative.

A possible question about the proposed approach is that in high dimensional space, it would be difficult to obtain a complete list of the extreme points of the dual polyhedron. We therefore made another test. In the example above there are two extreme points. Now we drop one of them, say $w^{2}$, and only keep the extreme point $w^{1}$. Then the optimal cost of the corresponding SOC problem is -902.93 , which is almost the same as the case with complete set of extreme points (-902.89). However, if we only keep $w^{2}$, the model gives us minimal cost $-1146.56(27 \%$ worse than the best minimal cost), which is not so good. Hence, our observation appears to

${ }^{3}$ Our model extends the uncertainty set from the one in Ang et al. (2014) to

$$
\mathcal{F}:=\left\{\mathbb{P}: \mathbb{P}(\tilde{z} \in \Omega)=1, \mathbb{E}_{\mathbb{P}}\left(\tilde{z}_{j}\right) \leq \mu_{j}, \mathbb{E}_{\mathbb{P}}\left(\tilde{z}_{j}^{2}\right) \leq \eta_{j}, j=1, \ldots, l\right\}
$$

Hence, the dual form has one extra non-negativity constraint,

$$
v_{j} \geq 0, j=1, \ldots, l,
$$

compared with that in Ang et al.(2014). When we conduct the numerical experiment following the formulation in Ang et al.(2014), we add this non-negativity constraint to their original SOCP formulation. Therefore, different numerical result is obtained here (not -929.88 but -900.9) with exact same formulation method taken. 
suggest that by "choosing" a few "crucial extreme points" we could still find a good solution without having to include all of the extreme points. A further research topic is then how to progressively include the "good extreme points" into the crucial set.

\section{Conclusion}

By considering the worst case over a set of probability measures for which we only have first and second order moment information, we may obtain robust and computationally efficient models for finding a solution to a two-stage stochastic optimization problem. It is demonstrated through a numerical example that this idea may lead to a fast algorithm with non-conservative solutions to a two-stage linear stochastic program. Therefore the SOC model may greatly widen the applicability of the two-stage recourse model in decision-making under uncertainty.

\section{References}

[1] Anderson E. J., P. Nash. 1987. Linear Programming in Infinite-dimensional Spaces: Theory and Applications. Wiley, Singapore.

[2] Ang S.K., F. Meng, J. Sun. 2014. Two-stage stochastic linear programs with incomplete information on uncertainty. European Journal of Operational Research233(1) 16-22.

[3] Ben-Tal A., A. Nemirovski. 2002. Robust optimization - methodology and applications. Mathematical Programming Series B 92(3) 453-480.

[4] Bertsimas, D., X. V. Duan, K. Natarajan, C.-P. Teo. 2010. Model for minimax stochastic linear optimization problems with risk aversion. Mathematics of Operations Research $\mathbf{3 5}(3)$ 580-602.

[5] Bertsimas, D., R. Freund. 2000. Data, Models, and Decisions: The Fundamentals of Management Science. South-Western College Publishing, Cincinnati.

[6] Rockafellar R. T., R-J.B. Wets 1998. Variational Analysis. Springer-Verlag, Berlin.

[7] Sion, M. 1958. On general minimax theorems. Pacific Journal of Mathematics 8(1) 171-176. 